\title{
Novel candidate genes and regions for childhood apraxia of speech identified by array comparative genomic hybridization
}

\author{
Jennifer J.S. Laffin, PhD ${ }^{1,2}$, Gordana Raca, MD, PhD 3 , Craig A. Jackson, MS ${ }^{1,2}$, Edythe A. Strand, $\mathrm{PhD}^{4}$, \\ Kathy J. Jakielski, PhD', Lawrence D. Shriberg, PhD ${ }^{1,6}$
}

\begin{abstract}
Purpose: The goal of this study was to identify new candidate genes and genomic copy-number variations associated with a rare, severe, and persistent speech disorder termed childhood apraxia of speech. Childhood apraxia of speech is the speech disorder segregating with a mutation in FOXP2 in a multigenerational London pedigree widely studied for its role in the development of speech-language in humans.

Methods: A total of 24 participants who were suspected to have childhood apraxia of speech were assessed using a comprehensive protocol that samples speech in challenging contexts. All participants met clinical-research criteria for childhood apraxia of speech. Array comparative genomic hybridization analyses were completed using a customized 385K Nimblegen array (Roche Nimblegen, Madison, WI) with increased coverage of genes and regions previously associated with childhood apraxia of speech.
\end{abstract}

Results: A total of 16 copy-number variations with potential consequences for speech-language development were detected in 12 or half of the 24 participants. The copy-number variations occurred on 10 chromosomes, 3 of which had two to four candidate regions. Several participants were identified with copy-number variations in two to three regions. In addition, one participant had a heterozygous FOXP2 mutation and a copy-number variation on chromosome 2, and one participant had a 16p11.2 microdeletion and copy-number variations on chromosomes 13 and 14 .

Conclusion: Findings support the likelihood of heterogeneous genomic pathways associated with childhood apraxia of speech.

Genet Med 2012:14(11):928-936

Key Words: 16p11.2; apraxia; dyspraxia; FOXP2; speech disorder

\section{INTRODUCTION}

Childhood apraxia of speech (CAS) is a rare, severe, and persistent speech sound disorder characterized by a deficit in planning/programming oral and laryngeal movements for speech. ${ }^{1}$ Speech motor profiles consistent with CAS appear to occur both as an idiopathic disorder limited to a core motor speech deficit and deficits in other speech processing domains, and in the context of complex neurodevelopmental disorders. In the latter context, CAS typically co-occurs with deficits in multiple domains, including intellectual disability, language impairment, nonverbal oral apraxia, dysarthria, and/or craniofacial and other dysmorphologies. ${ }^{2}$

The genetic origins of CAS are poorly understood. The most significant genomic finding to date is a mutation in the coding sequence affecting the forkhead box P2 (FOXP2) gene associated with CAS in approximately half of the widely cited multigenerational pedigree, the "KE" family. ${ }^{3-5}$ Reports of other affected individuals with sporadic and inherited translocations and disruptions affecting the FOXP2 locus have confirmed its role in speech and language impairment. ${ }^{6-10}$ FOXP2 is also the continuing focus of a large number of studies and discussions on the evolutionary biology of speech-language in humans. ${ }^{11,12}$ Recent findings have reported a speech disorder consistent with CAS in several complex neurodevelopmental disorders, including galactosemia ${ }^{13}$ and rolandic epilepsy. ${ }^{14}$

Identification of genes or loci that confer risk for CAS has been hampered by the low prevalence of CAS, the complexity of the phenotype, and the lack of a diagnostically conclusive assessment protocol. Although the latter two constraints have prohibited point and period prevalence estimates of idiopathic CAS, there is clinical consensus that CAS likely meets the criterion prevalence rate for a rare disorder in the United States of $\sim 1 / 1,500 .{ }^{15}$ The goal of our report was to identify candidate causal genes or regions of interest in 24 well-characterized participants with idiopathic CAS using custom array comparative genomic hybridization analysis (aCGH). aCGH has not been used to date in studies of the genomic origins of pediatric motor speech disorders. Although not as comprehensive as whole-genome or whole-exome sequencing, aCGH is a well-established wholegenome-analysis method for initial study of nonsyndromic intellectual and developmental disabilities. As described, the

\footnotetext{
${ }^{1}$ University of Wisconsin-Madison, Madison, Wisconsin, USA; ${ }^{2}$ Wisconsin State Laboratory of Hygiene, Madison, Wisconsin, USA; ${ }^{3}$ University of Chicago, Chicago, Illinois, USA; ${ }^{4}$ Mayo Clinic, Rochester, Minnesota, USA; ${ }^{5}$ Augustana College, Rock Island, Illinois, USA; ${ }^{6}$ Waisman Center, Madison, Wisconsin, USA. Correspondence: Lawrence D. Shriberg (shriberg@waisman.wisc.edu)
} 
increased coverage of regions associated with CAS provided the sensitivity to identify smaller potential copy-number variants (CNVs) within these regions (i.e., $>1-\mathrm{kb}$ as opposed to $>100-\mathrm{kb}$ gains or losses).

\section{MATERIALS AND METHODS}

\section{Participants}

Participants were recruited and consented for a study of pediatric motor speech disorders approved by institutional review boards at the data collection and data analyses institutions. All participants carried the diagnosis of CAS or suspected CAS from referring clinicians. The participants were assessed by one of two examiners using the Madison Speech Assessment Protocol, a 2-h protocol developed for research in speech sound disorders across the lifespan, including CAS. ${ }^{16}$ The Madison Speech Assessment Protocol includes 15 measures that provide a range of speaking conditions for age-sex standardized scores that profile a speaker's speech processing and speech production competence, precision, and stability. Digital recordings of responses to the Madison Speech Assessment Protocol speech tasks were processed using computer-aided methods for perceptual and acoustic analyses. Construct and concurrent validation studies have supported the diagnostic accuracy of four speech and prosodic signs to identify CAS across developmental periods (ref. 17 and unpublished data). All 24 participants were positive on at least three of the four signs of CAS recently validated as a behavioral marker of CAS. One of the four signs indexes transcoding (planning/programming) deficits in speech processing and the other three are acoustic-perceptual signs of deficits in phrasing, rate, and linguistic stress (unpublished data).

The Madison Speech Assessment Protocol also includes measures of intellectual function, receptive and expressive language, oral mechanism structure and function, oral-nonverbal motor function, and parental information on a participant's developmental, educational, and behavioral histories.

Table 1 includes individual descriptive information for 12 of the 24 participants with genetic findings plausibly associated with CAS (to be described) and summarized information for the remaining 12 participants with noninformative aCGH

Table 1 Phenotype data for 12 participants with childhood apraxia of speech (CAS) and informative aCGH findings and summary data for 12 participants with CAS and noninformative aCGH findings

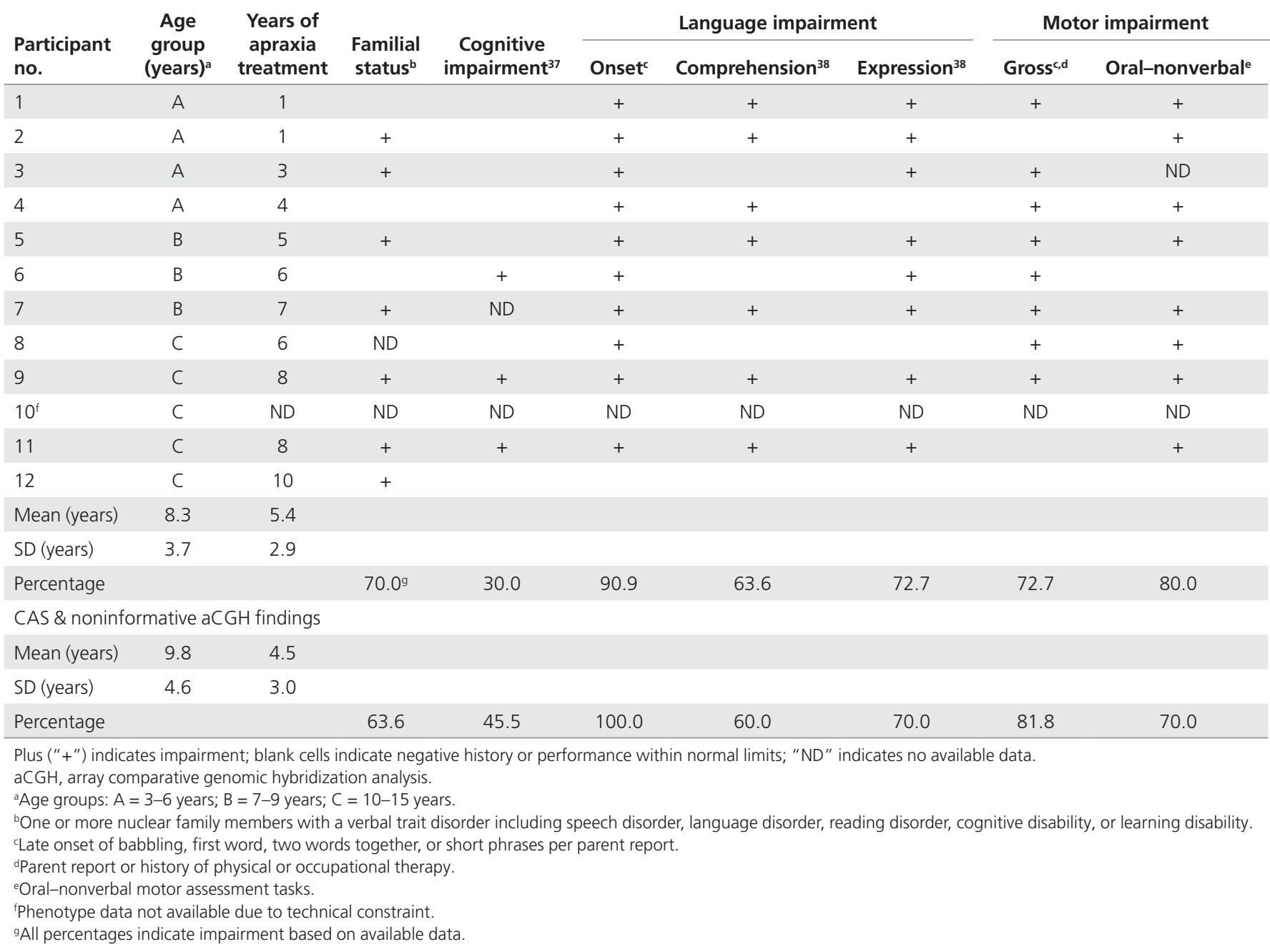


findings. Individual participant data for the informative group in Table 1 is aggregated in developmental groups (preschool, early elementary, adolescence) without sex status information to maintain anonymity.

Beginning with the individual participant data in the informative group, participants had a 2:1 male:female ratio, consistent with sex ratios reported in the idiopathic CAS literature. ${ }^{1}$ Consistent with findings indicating that CAS is a persistent disorder even with treatment meeting the standard of care, ${ }^{1}$ individual participants in the informative group had been receiving speech services for CAS for as long as 10 years. The $70 \%$ familial aggregation rate, coded as positive if the proband had at least one other biological family member with any type of speech sound disorder and adjusted for missing data, is appreciably higher than the $56 \%$ familial aggregation rate estimated for children with speech delay, the most prevalent class of speech sound disorders. According to the parent informants, only 1 of the 24 probands in this subsample of a larger study group had another nuclear family member with a clinical diagnosis of CAS.

The profiles of cognitive, language, and motor impairment scores of the 12 participants were similar to those summarized in a technical report on CAS. ${ }^{1}$ Adjusted for missing data, $30 \%$ of the participants had an intellectual disability, $91 \%$ were delayed in the onset of speech-language, $64 \%$ had impairments in language comprehension, and $73 \%$ had impairments in language expression. Last, 73 and $80 \%$ of participants, respectively, had impairments in gross motor and oral-nonverbal movements. These behavioral profiles of participants with CAS are consistent with the perspective that their processing constraint in planning/programming the articulatory gestures for speech is appropriately viewed as the signature deficit in what is otherwise a multiple-domain disorder. ${ }^{17}$

Crucially for the goals of our study, the summarized data were similar for participants with and without CNVs detected by array testing. As shown in Table 1, the summarized data for the two groups indicates similar average age and years of treatment and approximately similar (within 15 points) percentages of participants with positive findings on the seven behavioral measures. Additional analyses indicated that the two groups had approximately similar phenotypes indexing severity of CAS.

\section{FOXP2 sequencing}

All 24 participants evaluated by array were also evaluated for FOXP2 mutation status, by sequencing each of the seventeen FOXP2 coding exons (NCBI reference sequence: NM_014491.3). The exons were PCR amplified (AmpliTaq Gold PCR Master mix; Applied Biosystems, Carlsbad, CA) using oligos listed (Supplementary Table S1, online). PCR amplification and amplicon size were verified by gel electrophoresis. Sequences of each PCR amplicon were generated in both forward- and reverse-direction sequencing reactions with Big Dye Terminator v 3.1 (Applied Biosystems), purified with AxyPrep Mag DyeClean beads (Axygen Biosciences, Union City, CA), and run using either an ABI $3730 \times 1$ or $3130 \times 1$. Exon 15 of participant 5 was sequenced in triplicate for confirmation of a heterozygous change to be described. DNASTAR SeqMan Pro v 9.1.1, Polyphen-2 (version 2.2.2), and UCSC genome browser NCBI36/hg18 (tracks: dbSNP build 135), HGDP Allele Freq (Human Genome Diversity Project), HapMap, DGV (Database of Genomic Variants), and Genome Variants (variant base calls from nine genomes) were used for data analysis and interpretation of variants.

\section{aCGH analyses}

Genomic DNA was purified using the Qiagen PureGene DNA extraction kit reagents (Qiagen, Valencia, CA). Subject DNA was labeled and cohybridized with sex mismatched labeled control genomic DNA (Promega, Madison, WI). Copy-number analysis was performed using a customized $385 \mathrm{~K}$ Nimblegen array (Roche Nimblegen) with increased coverage of genes and regions previously associated with CAS. Table 2 includes information on these areas, including 21 genes or regions of interest associated with CAS or language phenotypes associated with multiple-domain involvement (i.e., cognitive, language, motor).

Laboratory methods were performed according to manufacturer specifications. Data analysis was performed using CytoSure Interpret Software Version 3.4.3 (Oxford Gene Technologies, Begbroke, Oxfordshire, UK). Regions of benign $\mathrm{CNV}$, as reported by the Database of Genomic Variants, ISCA database (The International Standards for Cytogenomic Arrays Consortium), and CHOP CNV databases (Children's Hospital of Philadelphia), were excluded from the final results. No recurrent CNVs were introduced into the analysis by the custom design as evaluated across specimens with sex mismatched normal controls. The HG18 human genome build, NCBI build 36.1, was used in the analysis and mapping. Deletions and duplications were required to contain five contiguous probes. The log threshold factor for gains was set to 0.3 and that for losses was set to 0.6. Array validation studies for several specimens indicated that the incidence of CNVs for targeted regions did not differ from the incidence in the clinical whole-genome arrays.

\section{FOXP2 sequencing}

\section{RESULTS}

One participant, (Table 1, participant 5), was found to have a heterozygous mutation, c.1789A $>\mathrm{C}$ in exon 15 . This base substitution causes a missense mutation, $\mathrm{N} 597 \mathrm{H}$, in the $\mathrm{C}$ terminal of the protein, just outside of the forkhead domain (Supplementary Figure S1, online). There are no common SNPs identified in the region. This mutation has not been reported before in the literature. The PolyPhen-2 prediction/confidence scores were 0.995 and 0.795 for HumDiv and HumVar, respectively, suggesting this variant is likely to be pathogenic. ${ }^{18}$

\section{aCGH analyses}

Table 3 is a summary of findings for the 12 participants in Table 1 with CNVs. The 16 row-wise entries in Table 3 include $\mathrm{CNVs}$ with plausible neural consequences for cognitive, speech, 


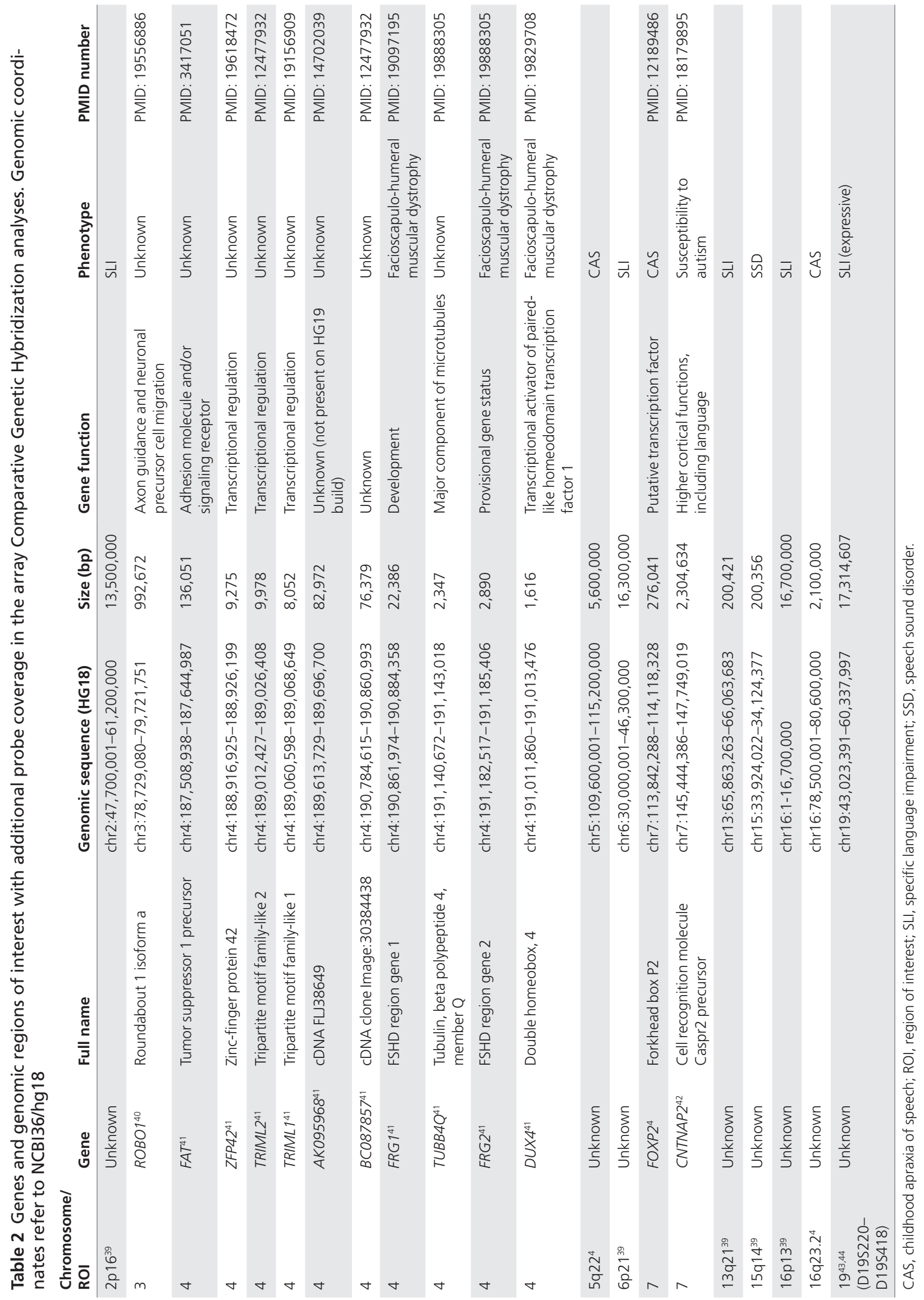




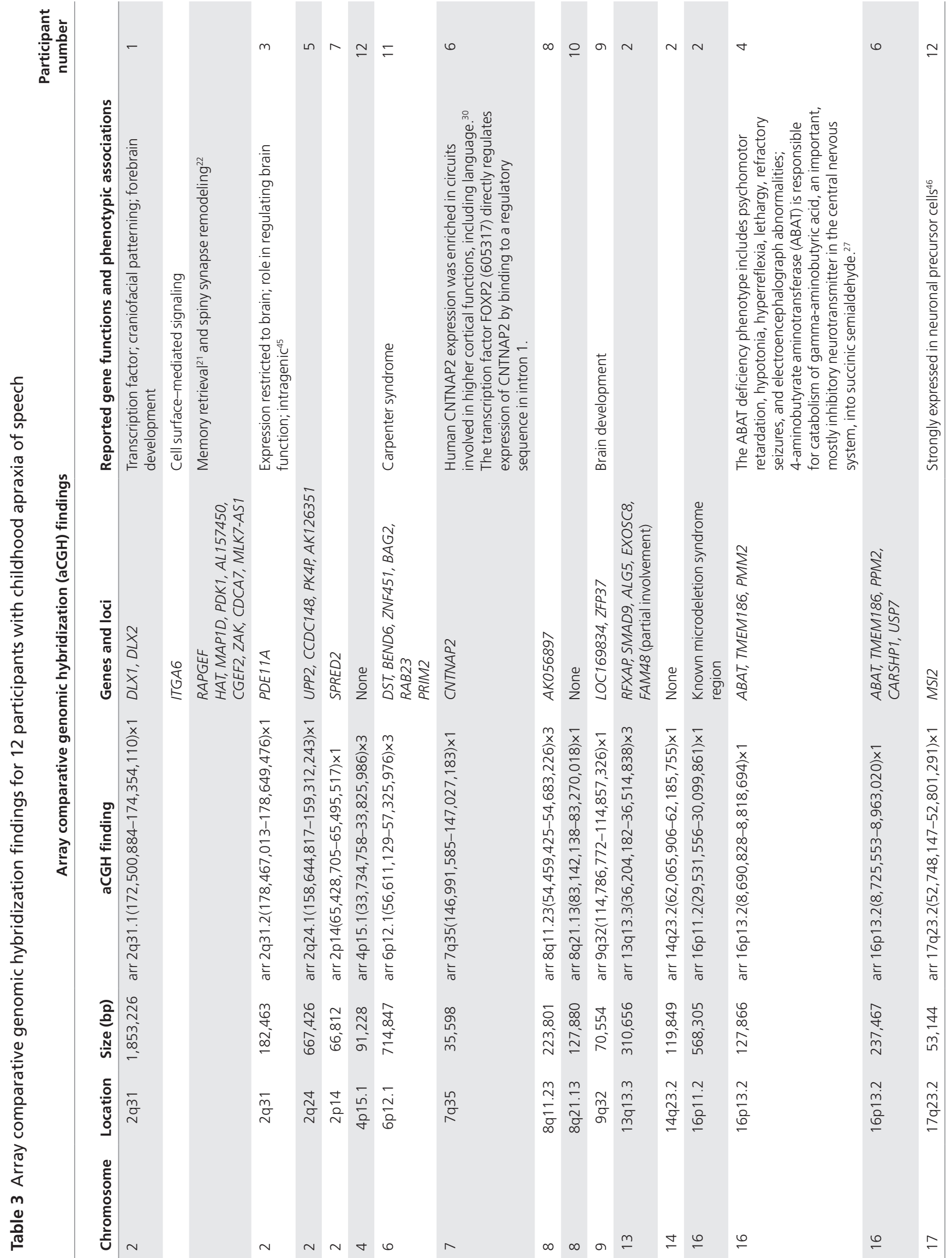


language, and motor processes in development and performance. The CNVs occurred on 10 chromosomes, three of which included two to four candidate regions: chromosome 2 (four regions), chromosomes 4, 6, 7, 8 (two regions), chromosomes $9,13,14,16$ (three regions), and chromosome 17 . The $16 \mathrm{CNVs}$ ranged in size from $\sim 36 \mathrm{~kb}$ to $1.8 \mathrm{Mb}$. As shown in the rightmost columns in Table 3, three participants had more than one CNV: participant 2 (3 regions), participant 6 (2 regions), and participant 12 ( 2 regions). Two of the participants in Table 1 had CNVs that included deletions of the same gene or genes. It is efficient to position additional information and comment on these and other findings in Table 3 in the following section.

\section{DISCUSSION}

\section{Chromosome 2}

As shown in Table 3, four deletions were identified on chromosome 2, including CNVs at 2p14, 2q24.1, 2q31.1, and 2q31.2.

\section{$2 \mathrm{p} 14$}

A deletion of $\sim 67 \mathrm{~kb}$ at $2 \mathrm{p} 14$ was detected as the single CNV in participant 7. This deletion eliminates a portion of SPRED2, a regulator of differentiation via the MAP kinase cascade. ${ }^{19,20}$ Two deletions have been reported in this region in the ISCA and Decipher databases, but both are much larger (2.2 and 4 $\mathrm{Mb})$ than the deletion identified in participant 7 . However, based on the function of the affected gene in differentiation of neuronal cells, ${ }^{19}$ the $67 \mathrm{~kb}$ deletion in this participant plausibly affects speech processing.

\section{$2 q 24.1$}

A deletion of $\sim 667 \mathrm{~kb}$ at $2 \mathrm{q} 24.1$ (participant 5) involves the UPP2, CCDC148, PK4P, and AK126351 (uncharacterized) genes. This participant was also found to have a heterozygous, likely pathogenic, FOXP2 mutation. The ISCA and Decipher databases report six deletions in this region, all greater than 2.3 Mb. Of particular interest is the phenotypic description of Decipher individual 254867 with a $2.3-\mathrm{Mb}$ deletion. This individual is reported to have speech delay, microcephaly, and intellectual and developmental disabilities, as well as tall stature. This deletion overlaps findings for the participant in the present database by $\sim 170 \mathrm{~kb}$ and involves the UPP 2 and CCDC148 genes. CCDC148 is a putative transcriptional modulator. Additional phenotype evaluation will be necessary to determine the contributory significance of the $\mathrm{CNV}$, if any, to the FOXP2-associated phenotype.

\section{$2 q 31.1$}

A $1.8 \mathrm{Mb}$ deletion at $2 \mathrm{q} 31.1$, the largest deletion detected in this patient cohort, contains the DLX1 and DLX2 genes, which belong to a family of transcription factors involved in craniofacial patterning and forebrain development. RAPGEF4, also within this deletion, is involved in memory retrieval and spiny synapse remodeling. ${ }^{21,22}$ RAPGEF4 is reported as a putative target for activation by FOXP $2,{ }^{23}$ as well as an autism susceptibility gene. ${ }^{24}$ Other genes in this region include HAT1, MAP1D,
ITGA6 (cell surface-mediated signaling), PDK1, AL157450 (hypothetical gene), CGEF2, ZAK, CDCA7, and MLK7-AS1. Deletions overlapping this region have been reported in the Decipher and ISCA databases, but all are much larger and associated with a more severe phenotype including intellectual and developmental disabilities as well as multiple congenital anomalies.

\section{$2 q 31.2$}

A 2 q31.2 deletion in participant 3 includes $\sim 182 \mathrm{~kb}$, deleting several exons of the PDE11A gene. Mutations in this gene have been reported in association with the autosomal dominant disorder pigmented nodular adrenocortical disease. A deletion of $245 \mathrm{~kb}$ affecting PDE11A has been reported in the ISCA database; however, it was described as a finding of unknown significance. All other reported deletions within this region are substantially larger.

\section{Chromosome 6}

The duplication of $\sim 715 \mathrm{~kb}$ identified at $6 \mathrm{p} 12.1$ in participant 11 overlaps with two reported duplications-a $15-\mathrm{Mb}$ duplication reported as pathogenic and a 793-kb duplication reported as of uncertain significance (ISCA database; pathogenic CNVs, and uncertain CNVs 19 January 2011). The smaller duplication of uncertain significance reported a phenotype of developmental delay and hypotonia. Hypotonia was also reported in the case history information by parental report for 2 of the 12 participants, but not for participant 11 . This region contains the DST, BEND6, ZNF451, BAG2, RAB23, and PRIM2 genes. $D S T$ encodes an adhesion junction plaque protein involved in anchoring neural intermediate filaments to the actin cytoskeleton. Homozygous loss of this gene produces a progressive neuropathy in mouse models. ${ }^{25} \mathrm{RAB} 23$ is a negative regulator of Sonic hedgehog signaling. Expression of RAB23 is high in spinal cord, somites, limb buds, and cranial mesenchyme in the developing mouse embryo. Adult mice show high levels of expression in the brain, heart, and lung. ${ }^{26} \mathrm{RAB} 23$ is also associated with recessive Carpenter syndrome characterized by distinctive skeletal anomalies and other congenital anomalies with no mention of speech disorder (OMIM no. 201000). ${ }^{27}$ It is unclear how duplication of these genes might be associated with the neurodevelopmental substrates of CAS.

\section{Chromosome 7}

Among the notable findings of this study is the identification of a 35-kb deletion within intron 13 of the CNTNAP2 gene. CNTNAP2 has become of wide-ranging interest in emerging studies reporting its association with a number of complex neurodevelopmental disorders including autism, language impairment, speech delay, dyslexia, and CAS. ${ }^{28}$ Of note, this gene, reported to be associated with a number of cortical functions, is regulated by the FOXP2 transcription factor. ${ }^{29,30}$ Although the detected deletion maps within the noncoding portion of the gene (intron 13), it could affect regulatory elements important for CNTNAP2 expression. A challenging finding is that this 
variant was identified in participant 6 , who, as discussed subsequently, also has a deletion in 16p13.2 that includes $A B A T$, TMEM186, and PPM2.

The FOXP2 sequencing findings for participant 8 described previously will be addressed in a separate report that integrates speech findings for this participant with those reported for two other families with FOXP2 mutations and affected members of the KE family. ${ }^{7,9}$ As reported next, this participant also had a deletion on chromosome 8 detected by array.

\section{Chromosome 8}

Two CNVs, a 223-kb duplication at 8q11.23 and a 128-kb deletion at $8 \mathrm{q} 21.13$, were identified in Participants 8 and 10 . Neither region contains a validated gene and all reported CNVs are substantially larger than those identified in these participants, leaving uncertain the significance of these findings. Additional phenotyping of participant 8 as well as in vitro and model organism studies will be necessary to define possible contributions to the CAS phenotype.

\section{Chromosome 9}

The deletion of $\sim 70.6 \mathrm{~kb}$ at $9 \mathrm{q} 32$ identified in participant 9 contains a hypothetical protein, LOC169834, as well as the zincfinger protein 37 . The zinc-finger protein has been implicated in chondrocyte differentiation as extrapolated from screening of a human fetal cartilage-specific cDNA library. ${ }^{31}$ It is a proposed candidate gene for Nager syndrome (acrofacial dysostosis), which is characterized by multiple congenital anomalies including skeletal anomalies, conductive hearing loss, and speech delay (OMIM no. 154400). ${ }^{27}$

\section{Chromosome 16}

Two findings on chromosome 16 are among the most significant for ongoing genomic research in CAS. Participant 4 and participant 6 had overlapping deletions at 16p13.2 that include the ABAT, TMEM186, and PMM2 genes. A disruption in the $A B A T$ gene is causally associated with autosomal recessive gamma-aminobutyric acid transaminase deficiency syndrome (OMIM no. 613163). ${ }^{27}$ TMEM186 is a transmembrane protein possibly associated with the mitochondria. PMM2 deficits are associated with congenital disorder of glycosylation type 1a, an autosomal recessive disorder. The disorders associated with disruptions in $A B A T$ and $P M M 2$ are characterized as severe; phenotypes have not been reported for carriers. It is possible that haploinsufficiency of these genes individually would not be sufficient for a CAS phenotype, but the contiguous deletion could have an additive effect possibly sufficient for CAS. Participant 6 also has a $35-\mathrm{kb}$ deletion in the region that includes CNTNAP2. The deletion in CNTNAP2 may be the causative factor, with the 16p13.3 deletion being a rare variant. Haploinsufficiency of these genes individually may have an associated phenotype, but it has not been fully characterized in carriers. Additional functional and inheritance studies would be necessary to define the phenotypic effects of these deletions. This study did not include parental DNA to determine inheritance.
The second notable finding for chromosome 16 was for participant 2. Participant 2 has three CNVs, including a 13q13.3 duplication, a 14q3.2 deletion, and a 16p11.2 microdeletion. The 13q13.3-duplication includes RFXAP, SMAD9, ALG5, EXOSC8, and a partial deletion of FAM48. The 14q23.3 deletion contains no known genes. Of note, participant 2 also has a 16p11.2 microdeletion, a widely discussed, prevalent deletion recently characterized as the 16p11.2 microdeletion syndrome. As discussed elsewhere, the history and behavioral profiles of this participant and another with CAS are consistent with the emerging literature on the 16p11.2 microdeletion syndrome and extend the phenotype of this syndrome to include $\mathrm{CAS}^{32}$.

\section{Multiple CNVs}

The findings that three participants had more than one CNV, including the participant with the 16p11.2 microdeletion, are consistent with recent observations that carriers of microdeletions such as 16p11.2 show enrichment for "second-hit" CNVs. ${ }^{33}$ These authors suggest that a "two-hit hypothesis" might include the expectation of elevated, double-hit rates among pathogenic CNVs with clearly variable penetrance and expressivity. In this study, the hypothesis would predict that the three participants with multiple CNVs are at risk for expression of more heterogeneous CAS phenotypes due to the additional genomic modifiers. Such genotype-phenotype hypotheses are readily testable in CAS research with samples of sufficient size.

\section{Conclusion}

We hypothesized that as has been found for a number of complex neurodevelopmental disorders, rare CNVs in genomic DNA may be associated with increased risk for CAS. Procedures to identify CAS included a standardized assessment protocol and well-developed perceptual and acoustic diagnostic classification methods. Consistent with the hypothesis, whole-genome high-resolution oligo array comparative genomic hybridization studies in 24 participants with CAS identified one participant with a 16p11.2 microdeletion, two participants with overlapping deletions affecting genes on 16p13.2, and 10 participants with potentially pathogenic copy-number changes reported in the genetics literature to be associated with neural function or more directly with speech-language disorders. CNTNAP2 was the only gene given additional probe coverage in the customized aCGH chip that had a potentially pathogenic variant in our sample. DLX1, PDE11A, RAPGEF4, and ZFP37, as well as other genes with currently unknown function, were identified as additional strong candidate genes for CAS. Several of our identified genes had gene family members identified in other studies of verbal trait disorders. Gene families include $A L G, B A G, C C D C, C D C, E X O S C, M A P, P D E, R A B, T M E M$, and $Z F P{ }^{23,34}$ These families are ubiquitous and redundant in function, with systems studies needed to delineate their individual and interactive contributions to CAS. Our design did not include the genomic information required for follow-up segregation analyses. 
To summarize, our study findings underscore the genetic and biochemical complexity of pediatric motor speech disorders such as CAS, which historically were expected to be associated with monogenetic causal pathways with high attributable risk. On the contrary, identification of new genes and regions of interest is consistent with a trend recognizing the likelihood of complex gene-to-gene interactions underlying CAS in neurogenetic and complex neurodevelopmental disorders. ${ }^{35}$ For clinical needs, such perspectives on genetic heterogeneities impact the likelihood of informative diagnostic yields from singlegene assays for CAS in favor of high resolution, whole-genome approaches such as array comparative genomic hybridization and comprehensive genome sequencing. The findings also underscore the complex challenges of confounding molecular mechanisms in next-generation sequencing of complex neurodevelopmental disorders. ${ }^{36}$

\section{SUPPLEMENTARY MATERIAL}

Supplementary material is linked to the online version of the paper at http://www.nature.com/gim

\section{ACKNOWLEDGMENTS}

This work was supported by a grant from the National Institute on Deafness and Other Communicative Disorders (DC000496) to L.D.S. and a core grant from the National Institute of Health and Development (HD03352) to the Waisman Center. We thank each of the participants and their families and Leah Frater-Rubsam, Sheryl Hall, Heather Karlsson, Heather Lohmeier, Maureen McCormack, Jane McSweeny, Christie Tilkens, and the University of Wisconsin Biotechnology Sequencing Center for their contributions to this research.

\section{DISCLOSURE}

The authors declare no conflict of interest.

\section{REFERENCES}

1. American Speech-Language-Hearing Association (ASHA). Childhood apraxia of speech [Technical Report], 2007. http://www.asha.org/policy.

2. Shriberg LD. A neurodevelopmental framework for research in Childhood Apraxia of Speech. In: Maassen B, van Lieshout P (eds). Speech Motor Control: New Developments in Basic and Applied Research. Oxford University Press: Oxford, UK, 2010:259-270.

3. Hurst JA, Baraitser M, Auger E, Graham F, Norell S. An extended family with a dominantly inherited speech disorder. Dev Med Child Neurol 1990;32:352-355.

4. Lai CS, Fisher SE, Hurst JA, et al. The SPCH1 region on human 7q31: genomic characterization of the critical interval and localization of translocations associated with speech and language disorder. Am J Hum Genet 2000;67:357-368.

5. Lai CS, Fisher SE, Hurst JA, Vargha-Khadem F, Monaco AP. A forkheaddomain gene is mutated in a severe speech and language disorder. Nature 2001;413:519-523.

6. MacDermot KD, Bonora E, Sykes N, et al. Identification of FOXP2 truncation as a novel cause of developmental speech and language deficits. Am J Hum Genet 2005;76:1074-1080.

7. Shriberg LD, Ballard KJ, Tomblin JB, Duffy JR, Odell KH, Williams CA. Speech, prosody, and voice characteristics of a mother and daughter with a 7;13 translocation affecting FOXP2. J Speech Lang Hear Res 2006;49:500-525.

8. Zeesman S, Nowaczyk MJ, Teshima I, et al. Speech and language impairment and oromotor dyspraxia due to deletion of $7 q 31$ that involves FOXP2. Am J Med Genet A 2006;140:509-514.
9. Rice GM, Raca G, Jakielski KJ, et al. Phenotype of FOXP2 haploinsufficiency in a mother and son. Am J Hum Genet A 2012;158A:174-181.

10. Palka C, Alfonsi M, Mohn A, et al. Mosaic 7q31 deletion involving FOXP2 gene associated with language impairment. Pediatrics 2012;129:e183e188.

11. Ramus F, Fisher SE. Genetics of language. In: Gazzaniga MS (ed). The Cognitive Neurosciences, vol. IV. MIT Press: Cambridge, 2009:855-872.

12. Newbury DF, Monaco AP. Genetic advances in the study of speech and language disorders. Neuron 2010;68:309-320.

13. Shriberg LD, Potter NL, Strand EA. Prevalence and phenotype of childhood apraxia of speech in youth with galactosemia. J Speech Lang Hear Res 2011;54:487-519.

14. Pal DK, Li W, Clarke T, Lieberman P, Strug L. Pleiotropic effects of the $11 \mathrm{p} 13$ locus on developmental verbal dyspraxia and EEG centrotemporal sharp waves. Genes Brain Behav 2010;9:1004-1012.

15. Roubertoux PL, de Vries PJ. From molecules to behavior: lessons from the study of rare genetic disorders. Behav Genet 2011;41:341-348.

16. Shriberg LD, Fourakis M, Hall SD, et al. Extensions to the Speech Disorders Classification System (SDCS). Clin Linguist Phon 2010;24:795-824.

17. Shriberg LD, Lohmeier HL, Strand EA, Jakielski KJ. Encoding, memory, and transcoding deficits in Childhood Apraxia of Speech. Clin Linguist Phon 2012;26:445-482.

18. Adzhubei IA, Schmidt S, Peshkin L, et al. A method and server for predicting damaging missense mutations. Nat Methods 2010;7:248-249.

19. Wakioka T, Sasaki A, Kato R, et al. Spred is a Sprouty-related suppressor of Ras signalling. Nature 2001;412:647-651.

20. Nonami A, Kato R, Taniguchi K, et al. Spred-1 negatively regulates interleukin-3-mediated ERK/mitogen-activated protein (MAP) kinase activation in hematopoietic cells. J Biol Chem 2004;279:52543-52551.

21. Ostroveanu A, van der Zee EA, Eisel UL, Schmidt M, Nijholt IM. Exchange protein activated by cyclic AMP 2 (Epac2) plays a specific and time-limited role in memory retrieval. Hippocampus 2010;20:1018-1026.

22. Woolfrey KM, Srivastava DP, Photowala $H$, et al. Epac2 induces synapse remodeling and depression and its disease-associated forms alter spines. Nat Neurosci 2009; 12:1275-1284.

23. Vernes SC, Oliver PL, Spiteri E, et al. Foxp2 regulates gene networks implicated in neurite outgrowth in the developing brain. PLOS Genet 2011;7:e1002145.

24. Bacchelli E, Blasi F, Biondolillo M, et al.; International Molecular Genetic Study of Autism Consortium (IMGSAC). Screening of nine candidate genes for autism on chromosome $2 q$ reveals rare nonsynonymous variants in the CAMP-GEFIl gene. Mol Psychiatry 2003;8:916-924.

25. Tseng KW, Peng ML, Wen YC, Liu KJ, Chien CL. Neuronal degeneration in autonomic nervous system of Dystonia musculorum mice. J Biomed SCi 2011;18:9.

26. Eggenschwiler JT, Espinoza E, Anderson KV. Rab23 is an essential negative regulator of the mouse Sonic hedgehog signalling pathway. Nature 2001;412:194-198.

27. OMIM (Online Mendelian Inheritance in Man). Johns Hopkins University, Center for Medical Genetics: Baltimore, 1996. http://www3.ncbi.nlm.nih. gov/omim/.

28. Peter B, Raskind WH, Matsushita M, et al. Replication of CNTNAP2 association with nonword repetition and support for FOXP2 association with timed reading and motor activities in a dyslexia family sample. J Neurodev Disord 2011;3:39-49.

29. Abrahams BS, Tentler D, Perederiy JV, Oldham MC, Coppola G, Geschwind $\mathrm{DH}$. Genome-wide analyses of human perisylvian cerebral cortical patterning. Proc Natl Acad Sci USA 2007;104:17849-17854.

30. Vernes SC, Newbury DF, Abrahams BS, et al. A functional genetic link between distinct developmental language disorders. N Engl J Med 2008;359:2337-2345.

31. Dreyer SD, Zhou L, Machado MA, et al. Cloning, characterization, and chromosomal assignment of the human ortholog of murine Zfp-37, a candidate gene for Nager syndrome. Mamm Genome 1998;9:458-462.

32. Raca G. Baas BS, Kirmani S, et al. Childhood Apraxia of Speech (CAS) in two patients with 16p11.2 microdeletion syndrome. Eur J Hum Genet, in press.

33. Girirajan S, Rosenfeld JA, Cooper GM, et al. A recurrent 16p12.1 microdeletion supports a two-hit model for severe developmental delay. Nat Genet 2010;42:203-209.

34. Spiteri E, Konopka G, Coppola G, et al. Identification of the transcriptional targets of FOXP2, a gene linked to speech and language, in developing human brain. Am J Hum Genet 2007;81:1144-1157. 
35. Bishop DV. Overlaps between autism and language impairment: phenomimicry or shared etiology? Behav Genet 2010;40:618-629.

36. Poot M, van der Smagt JJ, Brilstra EH, Bourgeron T. Disentangling the myriad genomics of complex disorders, specifically focusing on autism, epilepsy, and schizophrenia. Cytogenet Genome Res 2011;135: 228-240.

37. Kaufman AS, Kaufman NL. Kaufman Brief Intelligence Test-Second Edition AGS Publishing: Circle Pines, MN, 2004.

38. Carrow-Woolfolk E. OWLS: Oral and Written Language Scales. AGS Publishing: Circle Pines, MN, 1995.

39. Caylak E. A review of association and linkage studies for genetical analyses of learning disorders. Am J Med Genet B Neuropsychiatr Genet 2007;144B:923-943.

40. Lewis BA, Shriberg LD, Freebairn LA, et al. The genetic bases of speech sound disorders: evidence from spoken and written language. J Speech Lang Hear Res 2006;49:1294-1312.

41. Shriberg LD, Jakielski KJ, El-Shanti H. Breakpoint localization using array$\mathrm{CGH}$ in three siblings with an unbalanced $4 \mathrm{q} ; 16 \mathrm{q}$ translocation and childhood apraxia of speech (CAS). Am J Med Genet A 2008;146A: 2227-2233.

42. Bakkaloglu B, O'Roak BJ, Louvi A, et al. Molecular cytogenetic analysis and resequencing of contactin associated protein-like 2 in autism spectrum disorders. Am J Hum Genet 2008;82:165-173.

43. SLI Consortium. A genomewide scan identifies two novel loci involved in specific language impairment. Am J Hum Genet 2002;70:384-398.

44. SLI Consortium (SLIC). Highly significant linkage to the SLI1 locus in an expanded sample of individuals affected by specific language impairment. Am J Hum Genet 2004;74:1225-1238.

45. Kelly MP, Logue SF, Brennan J, et al. Phosphodiesterase 11A in brain is enriched in ventral hippocampus and deletion causes psychiatric disease-related phenotypes. Proc Natl Acad Sci USA 2010;107: 8457-8462.

46. Sakakibara S, Nakamura Y, Yoshida T, et al. RNA-binding protein Musashi family: roles for CNS stem cells and a subpopulation of ependymal cells revealed by targeted disruption and antisense ablation. Proc Natl Acad Sci USA 2002;99:15194-15199. 